\title{
LA EDUCACIÓN EN TIEMPOS DE BICENTENARIO
}

\author{
Dra. Olga Gonzáles Sarmiento
}

\begin{abstract}
RESUMEN
La situación educativa de América Latina ha llevado a plantear metas educativas para orientar el desarrollo de la educación hasta el 2021. Apuntan a la búsqueda de elevar la calidad educativa considerando la preparación del estudiante en los centros educativos, la intervención docente, la investigación, la participación social, en una palabra, una educación en continuo avance.
\end{abstract}

\section{ABSTRACT}

The educational situation in Latin America has lead to consider educational goals to guide education development to 2021 . They seek to increase quality in education considering student training at schools, teacher's presence, research, social participation, in general a continuous education development.

\section{PALABRAS CLAVE}

Educación, meta educativa indicada, carrera, investigación.

\section{KEYWORDS}

Education, indicated educational goal, career, research.



e levanto en la mañana y, casi por inercia, enciendo la "tele", hay que estar enterados delo que sucede, dicen, sí pues, hay que saber qué está pasando en el país y fuera de él, si es posible.

Según el ranking 2011 de la revista especializada Doing Bussincss "el Perú es el mejor país para invertir en Latinoamérica" y..., agrega el conductor del noticiero "las previsiones de crecimiento para nuestra economía son del 6\% mínimo para este año y para el 2012". Bien, pienso, buenas noticias para empezar el día, crece el país y crecemos todos .

Luego, debo hacer un largo viaje hasta la universidad, la ruta es básicamente por zonas residenciales, supuestamente tranquilas, aunque ahora último ya no se sabe. En el camino, carros atiborrados de personas queluchan por llegar a tiempo .

De pronto, sube con dificultad al bus una anciana, es muy mayor, el cobrador pide "asiento reservado, asiento para la señora", la jovencita que se encuentra en el asiento reservado hace oídos sordos, luego de una pequeña discusión sobre que pagó su pasaje y iqué tal raza! se levanta a regañadientes. Felizmente, no es muy frecuente, pienso, por lo general, todos selevantan sin mayor problema y ceden el asiento.

Continúa el recorrido y no falta quien haya comido una mandarina a manera de desayuno y vaya arrojando las cáscaras por la ventanilla del micro, o quizás que alguien entre al carro con una cartera muy grande o una mochila y, sin fijarse, golpee la cabeza de otra persona que se encuentra sentada, quizás la persona reclame, y la joven o el joven continúe su camino como si nada, en ningún momento se excusa. 
Es la rutina de todos los días, ese es uno de los recorridos; . en otro, las situaciones se repiten, idénticas, con díferencías en el paisaje de fondo, el entorno ha cambiado, ahora ya no se trata de zonas tipo residencial o de negocios, sino lugares más bien pobres, donde se mezclan grandes centros comerciales (ioh la modernidad!) junto con avenidas atestadas de combís, custers, ómnibus, camiones de carga, buses ínterprovíncíales, con cantidades ingentes de basura y desperdicios arrojados, sin bolsas, cada cierto trecho.

Me acuerdo, entonces, de lo que pensé en la mañana "crece el país y crecemos todos...", ya no estoy tan segura de que sea así, o de que vaya a ser así, por algunas zonas se ve demasiada pobreza, demasiado atraso, demasiada tugurízación, ¿cómo convivimos tantísimas personas en la capital?, ¿¿cuántas de esas personas cuentan con los servicios mínimos en sus viviendas?, ¿¿cuentan con una vivienda?, ¿ será esteuno de los motivos de tanta violencia, de tan poco respeto a la vida y a la propiedad? Hay muchos niños por la calle, es "hora de colegio", pero ellos están allí, haciendo cualquier cosa (a veces, trabajando, a veces, buscando cómo sobrevivir), totalmente ajenos a la "modernidad", a sus posibilidades de mejora, totalmente ajenos al progreso y, por lo que se ve, totalmente ausentes, lejanos, marginales a la educación.

¿Así vivimos en tiempos de postmodernidad? Falta una década para celebrar, probablemente, con bombos y platillos, el bicentenario de nuestra independencia, ya empezarán los discursos, las ceremonias, los fuegos artificiales, la celebración, pero, ¿'somos realmente libres?, ¿puede ser libre una persona que no cuenta con lo básico, con lo mínimo para vivir dignamente?, ¿qué de libre tiene vivir en la calle, pernoctar en el quicio de una puerta, debajo de un puente, oliendo terokal para evadir una realidad tan dura?

Hace poco mc1os de un mes tuve que ir por la Universidad Nacional de Ingeniería, hasta ahora recuerdo a un anciano que se encontraba caminando a dos cuadras de la universidad cargando un pequeño atado que contenía, supongo, todas sus pertenencias en la vida, calculo que el paquete no sobrepasaría los dos kilos de peso, sin embargo, el señor era tan anciano y, probablemente, tan débil, que avanzaba dos o tres pasos y se detenía para descansar colocando el bulto en el suelo, tomaba aliento, y luego iniciaba toda la operación otra vez; más allá, un demente, casi sin ropa, simplemente sentado en el suelo, hablando consigo mismo ocon sus demonios interiores.
¿Somos realmente un país emergente?, ¿el gran tigre americano?, ¿aquel país en el que todos deberían invertir?, ¿por qué se permiten tales situaciones?, ¿̇a dónde se acude cuando se presencian estos hechos?, ¿a la municipalidad?, no hay una oficina para tales casos, dicen que no les compete, que tiene que llamarse a tal pero no están muy seguros de sí hay que dirigirse allí o a otro lugar, da policía?, lo siento, 11? hay efectivos, mucha delincuencia, hay prioridades, disculpe, muchas cosas por atender . No me queda muy claro, ¿una persona no es prioridad?, no lo sé, algo está fallando sí no nos damos cuenta, algo ha sucedido y hemos perdido la brújula o quizás nos hemos acostumbrado. Así es Lima, moderna, pobre, rica, tugurízada, violenta, desigual, injusta, emergente, cruel, ¿con cuál nos quedamos?, ¿quizás con la que nos tocó para vivir?

Sí nos preguntamos ¿qué hay que cambiar?, probablemente la respuesta sería imucho!, idemasíado!, demasiado para pobres esfuerzos individuales, pero bueno, por algo se debe empezar, ¿no? A veces, la pequeña suma de pequeños esfuerzos conjuntos nos confirma que la excepción hace la regla.

Quizás un buen comienzo sería cambiar la educación, pero allí hay otro caballito de batalla, uno que para nuestro país ha sido difícil de cabalgar hace ya demasiado tiempo, siempre con reformas de pocos años que no conducen a nada efectivo, pocos, pobres progresos, algunos mayores y mejores que otros, pero, en el fondo, nada verdaderamente sólido, de calidad.

Se nos viene a la mente, entonces, que desde que la educación se convirtió, para algunos, en un negocio, vivimos una especie de "fiebre" educativa, los jóvenes (los que pueden) terminan una carrera, o solo el bachillerato, y dejan pendiente el título para continuar estudios de maestría. Simultáneamente a la maestría, es probable que se hallen estudiando algún diplomado y, luego sin haber concluido los estudios, ya se encuentren considerando estudiar un doctorado. Hay que ganarle tiempo al tiempo, tener 26 años y, sí es posible, ya encontrarse estudiando un doctorado, no importa la calidad de la enseñanza, si los profesores enseñan o no, sí cumplen con el sílabo o no lo hacen, no importa, lo que interesa es si se aprueba y sífue con buena nota, lodemás, no.

Hay que ganar esta carrera al tiempo, estudiar una carrera por Internet o asistir a clases el menor tiempo posible, importa el L'.íploma, título, grado, y en esta carrera muy pocos de estos estudiantes quizás estén 
evaluando si debe haber calidad en el servicio educativo, como si el término calidad se encontrara desfasado, limitado solo a determinados campos pero no aleducativo.

Entonces, en esta pérdida de mucho y búsqueda de muy poco, quizás nos damos cuenta de que somos un país emergente, prometedor, con una riqueza cultural inmensa, con una gastronomía aclamada a nivel mundial y con muchos deseos de seguir creciendo, pero en este proceso debemos aceptar también que aún tenemos que lidiar con grandes dificultades y desigualdades en nuestro camino hacia el bicentenario de nuestra independencia.

Se trata, sin duda, de un problema con muchos factores, pero ¿por algo habrá que empezar, no?, quizás por iniciar con una reevaluación real, verdadera del servicio educativo que ofrecemos, un darnos cuenta que, cuando educamos estamos básicamente formando a una sociedad, sea nuestro alumno un niño de pocos años o un adulto que está empezando a estudiar una carrera a sus 45 o 50 años porque nunca pudo permitírselo, pero ahora lo hace, con gran sacrificio y cansancio, después de haber trabajado una larga jornada de ocho horas o más.

A diez años del bicentenario de nuestra independencia, estamos creciendo, es indudable, pero, muchas veces, de manera desordenada, inequitativa.

Una muestra de equidad es el servicio educativo que ofrecemos, debe ser de la mejor calidad posible. para todos, para el que pague mucho, poquito o nada, el costo no debería ser lo más importante, sino, quizás el tenerencuenta que, para esa persona enespecífico, ese período educativo sea una de las pocas oportunidades desuperación quesele presente.

Una vez alguien me dijo, qué se le puede hacer, pues, su madre es una prostituta y su padre está en Lurigancho, como si esa pequeña persona, por esas razones, no tuviera más remedio que seguir ese camino, no vale esforzarse por ella porque es como una especie de alumno de segunda clase, quizás,justo por esos motivos, esa niña/o merecería un mejor servicio educativo o que alguien le brindara la "otra" visión del mundo que le haga superarse y esperar algo mejor delavida.

Las cifras alentadoras, los niveles de confianza en nuestro país son, indudablemente, buenas noticias, solo que deben tener su correlato en aspectos enriquecedores que nos alcancen a todos, emprender una mejora educativa real en nuestro país sería un muy buen comienzo, pero no una reforma aparente, de papel, sino una reforma real, verdadera que vaya a través de todos los niveles educativos, desde los iniciales hasta los universitarios.

Entonces, surge una pregunta más, ¿qué generación de peruanos deseamos que celebren el Bicentenario de la Independencia?, aquellos que trafican, que disparan a quemarropa, que estafan, que lastiman a un niño de la manera más cruel, o tal vez aquellos otros que se conforman con una educación pobre, quizás a nivel de bachillerato, pero que corresponde, en papeles, a un doctorado, porque consideran que lo importante es tener documentos que certifiquen su paso por "altos estudios universitarios".

Cabría preguntarnos, además, ¿cómo deseamos vivir el crecimiento7, ¿cómo estamos entendiendo la post modernidad?, ¿qué nos puede enseñar aún la historia?, ¿qué es lo rescatable de nuestra sociedad?, ¿cómo queremos vivir el bicentenario?, ¿qué tipo de sociedad estamos formando para ese momento?, ¿cómo estamos pensando, diseñando, mejorando la educación?

Falta una década y ese tiempo no es mucho cuando hablamos del devenir de un país, pero, son diez años valiosos. Un posible buen comienzo de algo grande que podría estar dado por la propuesta: Metas Educativas 2021: La educación que queremos para la generación de los bicentenarios. El documento de la Organización de Estados Americanos para la Educación, la Ciencia y la Cultura (OEI) que, ante esta circunstancia especial en nuestro continente, ha propuesto un megaproyecto que busca el compromiso con la calidad educativa de los países que conforman la región y que se encuentran celebrando el bicentenario de su independencia desde el año 2009 hasta el 2021.

Se trata de una apuesta por el futuro, es una invitación a un esfuerzo solidario y a un compromiso colectivo de los países iberoamericanos, en un marco de integración cultural, histórica y educativa que se cimente en principios de unidad dentro de la diversidad.

Son once metas generales y veintiocho metas específicas (asociadas a 39 indicadores con sus respectivos niveles de logro), las que conforman este ambicioso programa educativo que fue aprobado en la Cumbre de Jefes de Estado y de Gobierno de los Países Iberoamericanosel pasado 2010. 
En esas reuniones se habló de los desafíos de la educación en Iberoaméríca, entre ellos, se consideró a la pobreza y a la desigualdad como los más importantes yurgentes.

Según las estimaciones de la Comisión Económica para América Latina y el Caribe (CEPAL), organismo dependiente de la Organización de las Naciones Unidas, responsable de promover el desarrollo económico y social de la región, los pobres en América Latina y el Caribe son 213 millones de personas, algo así como el 40,6\% de la población. No obstante, sí nos referimos a las personas que viven en pobreza extrema, la cantidad llega a 79 millones, casi el15\% dela población.

Por otro lado, el analfabetismo bordea los 34 millones de personas, lo que equivale a casi el $10 \%$ de la población. Junto a estas terribles cifras, tenemos que el 40\% de los jóvenes y adultos de la región, unos 110 millones, no han culminado sus estudios primarios.

Todas estas diferencias en las posibilidades educativas básicas, se encuentran inevitablemente, asociadas a un difícil acceso al mercado laboral y, por supuesto, a la obtención de escasos ingresos económicos.

Como todos sabemos, la pobreza y la desigualdad tienen, a su vez, muchos rostros: problemas de alimentación y salud, bajo rendimiento y/o abandono escolar, deficiente o inexistente preparación para el trabajo, etc.

Lo que me recuerda que alguna vez escuché que "la pobreza se hereda"; lamentablemente, parece ser que es cierto, se heredan rasgos genéticos, rasgos caracterológicos, rasgos de todo tipo..., pero también se hereda la pobreza.

\section{LAS METAS EDUCATIVAS}

Desde otra perspectiva, las mediciones estan cia rizadas que se han realizado a lo largo de los últimos años han mostrado que el déficit de aprendizaje de los estudiantes iberoamericanos en competencias básicas, tales como matemáticas y comprensión lectoras es preocupante, por decir lo menos.

La información más reciente recogida sobre resultados académicos de países de la región es la que ofrecen dos herramientas: el Programa para la Evaluación Internacional de Alumnos (PISA por su nombre en inglés, Programme far Internatíonal
Student Assessment) del año 2006, llevada a cabo por la Organización para la Cooperación y el Desarrollo Económico (OCDE); y el Segundo Estudio Regional Comparativo y Explicativo (SERCE), llevado a cabo por el Laboratorio Latinoamericano de Evaluación de la Calidad de la Educación (LLECE) de la UNESCO, también en 2006.

Dichas evaluaciones nos han mostrado un panorama bastante desalentador en lo que se refiere a la calidad del servicio educativo que se viene brindando a través de las diferentes instituciones creadas para tal fin; por consiguiente, se nos presenta una serie de retos en el campo educativo, muchos de ellos, bastante difíciles de enfrentar.

En este sentido, se nos ha propuesto a los países de América Latina y el Caribe una serie de Metas Educativas que orientarán la educación que brindemos en el lapso restante hasta el año 2021.

Dichas metas se han planteado en cuatro niveles: metas generales, metas específicas, indicadores y niveles de logro. Las metas generales, que son once, establecen objetivos amplios y, en base a ellas, se fijan las metas específicas, que son veintisiete, y son las que implican la selección de un elemento significativo de la meta general.

Con el fin de concretar cambios reales se han establecido, además, treinta y ocho indicadores que expresan el criterio que será utilizado para evaluar los avances en las metas.

Y si hablamos de metas, ¿̇hacia dónde se quiere dirigir América Latina mientras espera celebrar sus bicentenarios?

El documento ha subdividido la temática en una serie de aspectos, entre ellos los siguientes:

\section{GOBERNABILIDAD Y PARTICIPACIÓN SOCIAL}

Como sabemos, no depende solo de la educación el que se resuelvan problemas sociales, para que esto suceda, es necesario que se produzcan transformaciones en otros ámbitos de la sociedad; por otro lado, tampoco es posible que una sociedad tenga un desarrollo justo sin una educación equitativa para sus miembros, una educación verdaderamentede calidad. 


\section{META GENERAL PRIMERA}

REFORZAR Y AMPLIARLA PARTICIPACIÓN DE LA SOCIEDAD EN LA ACCIÓN EDUCADORA.

\section{META ESPECÍFICA}

Elevar la participación de los diferentes sectores sociales y su coordinación en proyectos educativos: familias, universidades y organizaciones públicas y privadas, sobre todo de aquellas relacionadas con servicios de salud y promoción del desarrollo económico, social y cultural.

\section{INDICADORI}

Número de proyectos en los que diferentes sectores sociales participan y que se aplican de forma integrada.

- Nivel de logro: Cada año aumenta el número de proyectos innovadores que se desarrollan de forma coordinada en un territorio (municipio, departamento, región) en los que participan varios sectores sociales.

\section{EDUCARENLADIVERSIDAD}

Sí hablamos de nuestro país, una de las maneras de definirlo es como un país multilíngüe y plurícultural, definición que a decir verdad, calzaría perfectamente con más deun paísiberoamericano.

Por tanto, la segunda meta está referída a que la educación del bicentenario debe tener en cuenta la diversidad cultural y lingüística de la región. Para este fin, se busca propiciar el desarrollo de un bilingüismo equilibrado y del orgullo de la propia identidad, a fin de que todas las etnias establezcan relaciones, desde un sentimiento de igualdad, con respecto a las demás.

Y en esta preocupación por la equidad y la inclusión se considera, indudablemente, la integración de alumnos connecesidades educativas especiales a fin de aportar, por medio de la educación, una experiencia enriquecedora que fomente la comprensión y la solidaridad.

\section{META GENERAL SEGUNDA}

LOGRAR LA IGUALDAD EDUCATIVA Y SUPERAR TODA FORMA DE DISCRIMINACIÓN EN LA EDUCACIÓN.

\section{META ESPECÍFICA}

Garantizar el acceso y la permanencia de todos los niños en el sistema educativo mediante la puesta en marcha de programas de apoyo y desarrollo de las familias para favorecer la permanencia de sus hijos en la escuela.

\section{INDICADOR2}

Porcentaje de familias con dificultades socio económicas que reciben apoyo para garantizar la asistencia habitual de sus hijos a las escuelas.

- Nivel de logro: En 2015, al menos el 30\% de las familias que se sitúan por debajo del umbral de pobreza recibe algún tipo de ayuda económica que garantiza el desarrollo integral de los niños y su asistencia a la escuela, y el $100 \%$ la recibe en el 2021.

\section{ATENCIÓN INTEGRAL DE LA PRIMERA INFANCIA}

Los hallazgos de la Neurociencia nos dicen que el desarrollo cerebral de los primeros años afecta tanto la salud mental y física como el comportamiento por el resto de la vida; por tal motivo, se ha de tener cuidado en integrar permanentemente la dimensión social y la dimensión educativa, debido a que lo que aprenden después los niños en la escuela, depende ampliamente de las competencias sociales, emocionales y cognitivas que hayan desarrollado en sus primeros años de vida.

\section{META GENERAL TERCERA}

AUMENTAR LA OFERTA DE EDUCACIÓN INICIAL Y POTENCIAR SU CARÁCTER EDUCATIVO .

\section{META ESPECÍFICA 6}

Aumentar la oferta de educación inicial para niños de 0a6años.

\section{INDICADORS}

Porcentaje de niños de $\mathrm{O}$ a 6 años que participan en programas educativos.

- Nivel de logro: En 2015 recibe atención educativa temprana entre el $50 \%$ y el $100 \%$ delos niños de 3a 6 años, y el100\% la recibe en 2021. 
En 2015, entre el 10\% y el 30\% de niños de O a 3 años participa en actividades educativas, y entre el 20\% y el50\% lo hace en 2021.

\section{GARANTIZAR EDUCACIÓN \\ EL ACCESO A LA}

Pobreza y analfabetismo van de la mano, es triste pero es verdad, para huir del círculo vicioso de la pobreza extrema es necesario que los niños reciban entre once y doce años de educación formal, a fin de que puedan contar con claras posibihdades para salir de la pobreza o para no caer en ella por tener como única opción el acceder a empleos precarios, inestables o con escasa remuneración.

Por tanto, los esfuerzos no deben descansar solamente en la ampliación de la cobertura educativa en sí misma, sino, sobre todo, en la creación de condiciones equitativas que garanticen el acceso de los niños y jóvenes al sistema educativo.

\section{METAGENERALCUARTA}

UNIVERSALIZAR LA EDUCACIÓN PRIMARIA Y LA SECUNDARIA BÁSICA, Y AMPLIAR EL ACCESO A LA EDUCACIÓN SECUNDARIA SUPERIOR.

\section{META ESPECÍFICA 8}

Asegurar la escolarización de todos los niños en la educación primaria y en la educación secundaria básica en condiciones satisfactorias.

\section{INDICADORIO}

Porcentaje de escolarización y de finalización de la educación primaria.

- Nivel de logro: En 2015, el 100\% del alumnado está escolarizado en educación primaria, y entre el $80 \%$ y el $100 \%$ la termina a la edad correspondiente. En 2021, más del 90\% de los alumnos termina la educación primaria a la edad establecida.

\section{INDICADORII}

Porcentaje ele escolarización y ele finalización de la educación secundaria básica.

- Nivel de logro: En 2015, entre el 60\% y el 95\% de los alumnos están escolarizados en educación secundaria básica, y entre el 70\% y el100\% lo está en 2021. Entre el 40\% y el 80\% del alumnado termina la educación secundaria básica en 2015, y entre el60\% y el90\% la concluye en 2021.

\section{APUESTA POR LA CALIDAD DE LA ENSEÑANZA}

La calidad educativa se halla estrechamente unida a la calidad del currículo que organiza las experiencias de aprendizaje que van a vivir los estudiantes, pero para que dichas experiencias resulten de provecho deben ser significativas, a la manera como lo denomina DavidAusubel(1983:18):

"Un aprendizaje es significativo cuando los contenidos son relacionados de modo no arbitrario y sustancial (no al pie de la letra) con lo que el alumno ya sabe. Por relación sustancial y no arbitraria se debe entender que las ideas se relacionan con algún aspecto existente específicamente relevante ele la estructura cognoscitiva del alumno, como una imagen, un símbolo ya significativo, un concepto o una proposición".

Pero ¿̇a qué se denomina currículo significativo? Un currículo significativo es aquel que busca conectar con los intereses de los alumnos y con sus formas de vida, que busca adaptarse a sus ritmos de aprendizaje, que establece de forma permanente la relación entre lo aprendido y las experiencias que los alumnos viven fuera de la escuela, en una palabra, un currículo que establece relación permanente con la realidad.

\section{META GENERAL QUINTA}

\section{MEJORAR LA CALIDAD DE LA EDUCACIÓN Y EL CURRÍCULO ESCOLAR .}

\section{METAESPECÍFICAIO}

Mejorar el nivel ele adquisición ele las competencias básicas y de los conocimientos fundamentales por parte de los alumnos.

\section{INDICADOR 13}

Porcentaje de alumnos con niveles satisfactorios ele logro en competencias básicas en las pruebas nacionales e internacionales.

- Nivel ele logro: Disminuye en al menos un 20\% el número de alumnos situados entre los dos niveles bajos de renclimiLnto en las pruebas de LLECE $6^{\circ}$ grado, PISA, TIMMS o PIRLS en las que 
participan diferentes países. Aumentan en la misma proporción los alumnos en los dos niveles altos en dichas pruebas.

\section{EDUCACIÓNTÉCNICO-PROFESIONAL}

Hoy en día es muy común ver a jóvenes que postulan a un número cada vez más creciente de universidades que, en algunos casos, continúan ofreciendo carreras que se encuentran saturadas para el mercado laboral existente .

Nosesueleinvestigarla oferta educativa enfuncióna losrequerimientos delmercado, entonces, se ofrecen a través de diversas vitrinas, las mismas certificaciones que no conducen realmente a la empleabilidad del futuro profesional.

Por tal motivo, resulta interesante la propuesta de una educación técnico-profesional que posibilite, en un campo diferente, mejores opciones de empleo.

\section{META GENERAL SEXTA}

FAVORECER LA CONEXIÓN ENTRE LA EDUCACIÓN Y EL EMPLEO A TRAVÉS DE LA EDUCACIÓN TÉC NICO -PROFESIONAL (ETP).

\section{META ESPECÍFICA 16}

Mejorar y adaptar el diseño de la educación técnicoprofesional de acuerdo con las demandas laborales.

\section{INDICADOR23}

Porcentaje de carreras técnico-profesionales cuyos currículos son diseñados por competencias teniendo en cuenta la demanda laboral.

Nivel de logro: En 2015, entre el 20\% y el 70\% de los centros de formación técnico-profesional organiza las carreras en función de las competencias derivadas de la demanda laboral, y entre el $50 \%$ y el100\% lo concreta en el 2021.

\section{INDICADOR 24}

Porcentaje de alumnos que realiza prácticas formativas en empresas.

- Nivel de logro: En 2015, entre el 30\% y el 70\% de los alumnos de educación técnico-profesional realiza prácticas en empresas o instituciones laborales, y entre el70\% yel100\% lo hace en 2021.

\section{ALFABETIZACIÓN Y EDUCACIÓN A LO LARGODELAVIDA}

Algunos de nuestros abuelos, en su época, se sintieron felices de poseer secundaria completa, la exigencia para nuestros padres fue la de acceder a estudios universitarios para llegar a cumplir el gran sueño de ser profesionales; luego, obtener el ansiado título no fue suficiente y había que proseguir con estudios de postgrado... Mucho han cambiado las exigencias, pero no solo en términos de certificaciones, también lo han hecho en el aspecto de que cada profesional debe seguir aprendiendo a lo largo de toda su vida, el famoso, long life learning, el continuar aprendiendo, en una palabra, el aprender a aprender.

\section{META GENERAL SÉPTIMA}

OFRECER A TODAS LAS PERSONAS OPORTUNIDADES DE EDUCACIÓN A LO LARGODETODALA VIDA

\section{META ESPECÍFICA 18}

Garantizar el acceso a la educación a las personas jóvenes y adultas con mayores desventajas y necesidades.

\section{INDICADOR26}

Porcentaje de población alfabetizada.

- Nivel de logro: Antes de 2015 la tasa de alfabetización en la región se sitúa por encima del $95 \%$.

\section{INDICADOR27}

Porcentaje de personas jóvenes y adultas recién alfabetizadas que continúa estudiando.

- Nivel de logro: Entre el 30\% y el 70\% de las personas jóvenes y adultas recién alfabetizadas continúa cursando estudios equivalentes a la educación básica.

\section{META ESPECÍFICA 19}

Incrementar la participación de los jóvenes y adultos en programas de formación continua presenciales y a distancia.

INDICADOR 28 
Porcentaje de jóvenes y adultos que participa en programas de formación y capacitación continua presenciales y a distancia .

- Nivel de logro: En 2015, el 10\% de las personas jóvenes y adultas participa en algún curso de formación, yel 20\% lo hace en 2021.

Sin embargo, lo que proponen estas metas educativas no se trata solo de lograr que los profesionales continúen especializándose o que las personas recién alfabetizadas lean y escriban, sino también que todas ellas alcancen las competencias propias de la educación y participen en programas que favorezcan su inserción laboral.

\section{DESARROLLO PROFESIONAL DE LOS DOCENTES}

Un conocido dicho popular afirma: "Nadie da lo que no tiene" y, como la experiencia nos ha enseñado, es verdad; por tal motivo, es imprescindible cuidar la calidad profesional de los docentes desde el período formativo, pero no solo en lo que se refiere al aspecto netamente académico, sino, sobre todo, en su formación como ser humano que va a colaborar en el crecimiento y mejora de otros seres humanos.

Sin embargo, no se trata solo de la formación docente, no podemos olvidar que el desarrollo profesional docente y la mejora de la escuela están estrechamente relacionados dado que los docentes trabajan en un contexto social y cultural determinado y en unas condiciones educativas ylaborales específicas.

Por tanto, las políticas públicas a favor del profesorado necesitan tener en cuenta estos contextos y condiciones para remover los posibles obstáculos que limitan el éxito de determinadas iniciativas orientadas de forma específica a su desarrollo profesional.

\section{METAGENERALOCTAVA}

\section{FORTALECER LA PROFESIÓN DOCENTE.}

\section{META ESPECÍFICA 20}

Mejorar la formación inicial del profesorado de primaria y de secundaria.

\section{INDICADOR 29}

Porcentaje de titulaciones de formación inicial docente con acreditación oficial de su calidad.
- Nivel de logro: En 2015 están acreditadas, al menos, entre el 20\% y el 50\% delas titulaciones de formación inicial, yentreel50\%yel100\% en 2021.

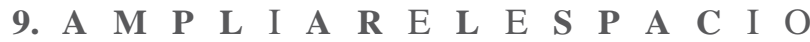
IBEROAMERICANO DEL CONOCIMIENTO Y FORTALECER LA INVESTIGACIÓN CIENTÍFICA

Nos encontramos en una sociedad que se denomina del Conocimiento, así, con mayúsculas, el conocimiento es un valor, tiene un costo, pero a la vez, es responsabilidad de todos, debemos crearlo, debemos compartirlo.

Los países del Bicentenario estamos en pleno crecimiento, pero este es desigual, ínequítatívo, e incluso, desordenado. Continuamos, en muchos casos, con una industria pobre, arcaica, que no ayuda al desarrollo aunque existen, innegablemente, loables esfuerzos individuales o de pequeños grupos, pero no hay una concertación, un plan común orientado aldesarrollo.

La universidad,como institución cuya razón de ser es la de crear conocimiento, debe investigar pues sí no se investiga, no se crea conocimiento y sí no se crea conocimiento, se mantiene el statu quo: no hay investigación, no hay conocimiento, no hay desarrollo.

\section{META GENERAL NOVENA}

\section{AMPLIAR EL ESPACIO IBEROAMERICANO DEL CONOCIMIENTO Y FORTALECER LA INVESTIGACIÓN CIENTÍFICA.}

\section{META ESPECÍFICA 22}

Apoyar la creación de redes universitarias para la oferta de postgrados, la movilidad de estudiantes e investigadores y la colaboración de investigadores iberoamericanos que trabajan fuera dela región.

\section{INDICADOR 32}

Porcentaje de becas de movilidad de los estudiantes e investigadoresentre los países iberoamericanos.

- Nivel de logro: En 2015, en toda la región, las becas de movilidad de estudiantes e investigadores alcanzan a 8.000, y a 20.000 en 2021. 


\section{INVERSIÓN EN EDUCACIÓN}

Invertir en educación es invertir en desarrollo, ningún país sale adelante si la mayoría de su población noseencuentra adecuadamente educada. En el idioma de la economía, a las personas se les denomina capital humano y ese capital humano se debe encontrar adecuadamente preparado.

Mínimamente debe comprender lo que lee, escribir con claridad y coherencia, sacar cuentas o tener una titulación a nivel superior que le haya enseñado a desarrollar habilidades mentales complejas, todo esto resulta imprescindible para mejorar la competitividadenla economía decualquier país.

\section{META GENERAL DÉCIMA}

INVERTIR MÁS E INVERTIR MEJOR.

\section{META ESPECÍFICA 24}

Aumentar el esfuerzo económico de cada país para el logro delas Metas Educativas 2021.

\section{INDICADOR 35}

Elaboración en el año 2010 de un plan de financiamiento por cada país para el logro delas metas y actualizarlo periódicamente.

- Nivel de logro: Se aprueba un plan en cada país, se evalúa y se adapta cada tres años.

\section{META ESPECÍFICA 25}

Incrementar la solidaridad internacional con los países que manifiestan mayores dificult ades.

\section{INDICADOR36}

Coordinar un Fondo Solidario para la Cohesión Educativa en el año 2011 con un plan de acción hasta 2021.

- Nivel de logro: Se desarrolla y coordina el Fondo Solidario, el cual aporta entre el $20 \%$ y el $40 \%$ de lo que comprometen los países y las regiones con mayor retraso educativo para el cumplimiento de las metas.

\section{EVALUAR EL FUNCIONAMIENTO DE LOS SISTEMAS EDUCATIVOS}

Si se habla de educar, potenciar al capital humano, evidentemente, se habla de educación, volvemos, por tanto, a lo tratado anteriormente, es necesaria una buena educación, una educación de calidad, pero para todos, así, los sistemas educativos deben ser evaluados permanentemente pero sin que intervengan preferencias de ningún tipo que no sean las estrictamenteeducativas.

Entonces,en esta década se debe evaluar y reevaluar el nivel de cumplimiento de las metas educativas como país que se dirige hacia el bicentenario de su independencia.

\section{LAS METAS EDUCATIVAS, SUS INDICADORES Y SUS NIVELES DE LOGRO}

\section{META GENERAL DÉCIMO PRIMERA}

EVALUAR El FUNCIONAMIENTO DE LOS SISTEMAS EDUCATIVOS Y DEL PROYECTO "METAS EDUCATIVAS 2021".

\section{META ESPECÍFICA 26}

Fortalecer los sistemas de evaluación de cada uno de los países.

\section{INDICADOR 37}

Reforzar los institutos de evaluación, los sistemas de planificación y las unidades de estadística de los países.

- Nivel de logro: En 2015 todos los países han consolidado sus institutos de evaluación y las unidades de planificación y de esta dística.

\section{META ESPECÍFICA 27}

Asegurar el seguimiento y la evaluación del proyecto Metas Educativas 2021.

\section{INDICADOR38}

Crear el Instituto de Seguimiento y Evaluación de las Metas Educativas y su Consejo Rector, en el que participen los representantes de los sistemas de evaluación delos países.

- Nivel de logro: El Instituto de Seguimiento y Evaluación de las Metas Educativas presenta ,cada dos años y por país, un informe general sobre el cumplimiento delasmetas.

\section{META ESPECÍFICA 28}

Fortalecer la participación de los distintos sectores sociales en el desarrollo y en la supervisión del proyecto Metas Educativas 2021. 


\section{INDICADOR39}

Crear el Consejo Asesor de las Metas Educativas 2021.

- Nivel de logro: El Consejo elabora, al menos, un informe cada dos años sobre el desarrollo del proyecto MetasEducativas 2021.

Como vemos, se trata de una propuesta interesante y ambiciosa, cada país de Iberoamérica se encuentra, en estos momentos, trabajando por el logro de las Metas Educativas 2021, a manera de preparación a la mejor celebración que podría darse en el bicentenario de su independencia, no solo compuesta por programas alusivos, marchas, discursos, fuegos artificiales, sino, el mejor regalo que se le puede dar a una sociedad, la posibilidad de desarrollarse de manera equitativa y justa por medio dela educación de sus ciudadanos.

Dra. Oiga Gonzáles Sarmiento

Doctora en Educación de la Universidad Femenina del Sagrado Corazón. Actualmente se encuentra desempeñando como docente e investigadora en la UNIFÉ. 